\title{
CALCULATION OF CLIMATE LOADS DESIGN VALUES ACCORDING TO THE PROBABILITY MODEL OF ANNUAL MAXIMUM SERIES
}

Scientific paper

(Received: 19 April 2021; accepted: 19 November 2021)

\section{Mykola Pashynskyi}

Department of building, road machines and construction, Central Ukrainian National Technical University, Kropyvnytskyi, Ukraine, PhD, Senior lecturer

Corresponding author: filonalone@gmail.com

\section{Victor Pashynskyi}

Department of building, road machines and construction, Central Ukrainian National Technical University, Kropyvnytskyi, Ukraine, Sc. D., Professor

\section{Evgeniy Klymenko}

Department of reinforced concrete constructions and transport constructions, Odessa State Academy of Civil Engineering and Architecture, Odessa, Ukraine, Sc. D., Professor

\begin{abstract}
The aim of this work is to improve a method for determining the characteristic values of climatic loads according to a probabilistic model of the annual maxima sequence, by choosing a rational type of generalized extreme value distribution law. An analysis is provided regarding the suitability of using four types of distributions for describing a data collection of maximum values of climatic loads. Using example data from the meteorological stations of Ukraine, it is found that for coefficients of variation smaller than $0.85-1.0$, it is advisable to use the double exponential Gumbel distribution (generalized extreme value distribution type-I), and at higher values of the coefficient of variation, it is advisable to use the Weibull distribution (generalized extreme value distribution typeIII). Recommendations are provided for considering the accuracy in the estimations of the characteristic values of loads according to the probabilistic model for the annual maximum value series.
\end{abstract}

Keywords: Annual maxima; characteristic values; climatic loads; probabilistic description. 


\section{INTRODUCTION}

Climatic loads and their impacts represent some of the most variable elements considered during the design of construction projects. Their values are mainly determined by the climatic conditions and the geographical location of the construction site [1-3]. For the design of load-bearing building structures based on the semi-probabilistic method of limit states according to the requirements of the Eurocodes and the State Building Standards of Ukraine, characteristic and design values of the loads are generally used. The design values of the climatic loads are usually set depending on the return period. The characteristic values are set to be exceeded only once every 50 years.

The traditional method for determining the design and characteristic values of climatic loads [1-3] is to describe the results of meteorological observations with a probabilistic model of the sequence of maximum values distributed by the double exponential Gumbel distribution [4]. This approach is widely used to standardize snow and wind loads [2-7]. Notably, other types of extreme values distributions can be used, as shown in other works [8-10]. The possibility of describing distributions of maximum load values by using distributions of various types has been analyzed in the works [8,11], among others.

The main disadvantage of the Gumbel distribution lies in the infinite range of its function, which does not correspond to the fundamentally positive areas for determining the loads on structures. The large coefficients of variation lead to a significant probability of physically impossible negative values of loads, leading to errors in the determination of the characteristic values.

In addition, another important concern is the accuracy of the estimation of the characteristic values of the load according to the data collection of annual maxima; this accuracy depends on the duration of the meteorological observations and characteristics of the annual maxima distribution. Resolving this issue will allow researchers to determine the accuracy when estimating the characteristic values of climatic loads, even when using different distribution laws to describe the annual maxima.

The above specifics regarding using the probabilistic model of the sequences of annual maxima for the description of climatic loads have encouraged the further improvement of the methodology for the determination of the characteristic values, as the accuracy and reliability of the determination of the loads significantly affect the reliability and economic value of construction projects being designed or already in use [12].

The purpose of this work is to improve the method for determining the characteristic values of climatic loads according to the probabilistic model of the of annual maxima sequence, based on a reasonable choice of a rational type of generalized extreme value distribution. Solving these problems will increase the accuracy and reliability of the determination of the characteristic values of the loads, based on the appropriate probabilistic model of the maximum value sequence.

\section{DISTRIBUTIONS OF EXTREMAL VALUES AND METHODS OF THEIR ANALYSIS}

Four types of distributions of extreme values are analyzed. The first three are described and examined in the Emil J. Gumbel monograph [4], and the truncated distribution of the Gumbel is a modification of the distribution of the first type. The following notations of these distributions are based on the recommendations [2], as more convenient for practical use.

The generalized extreme value distribution type-I (double exponential Gumbel distribution) has an integral distribution function and probability density function, as follows:

$$
\begin{aligned}
& F(Q)=\exp \left[-\exp \left(\frac{\alpha-q}{\beta}\right)\right] \\
& f(Q)=\frac{1}{\beta} \exp \left[\frac{\alpha-Q}{\beta}-\exp \left(\frac{\alpha-q}{\beta}\right)\right]
\end{aligned}
$$

The parameters $\alpha$ and $\beta$ are determined based on the mean value $M$ and standard deviation $S$ of an existing data collection of maximum values according to the methodology $[2,4]$. The design load value corresponding to the return period $T$ is calculated as follows:

Pashynskyi, M, Pashynskyi, V, Klymenko, E 


$$
Q(\mathrm{~T})=\alpha-\beta \cdot \ln [-\ln (1-1 / T)]
$$

The Gumbel distribution has a constant value of skewness of $A=1.14$, and its form is determined by the mean value and standard deviation. Changes in the appearance of the Gumbel double exponential distribution with the expected value $M=100$ depending on its coefficient of variation are shown in Fig. 1. It can be seen from the figure that with small values of the coefficient of variation, the probability of negative values is quite small. With the growth of the coefficient of variation, this probability increases, as shown in Table 1.

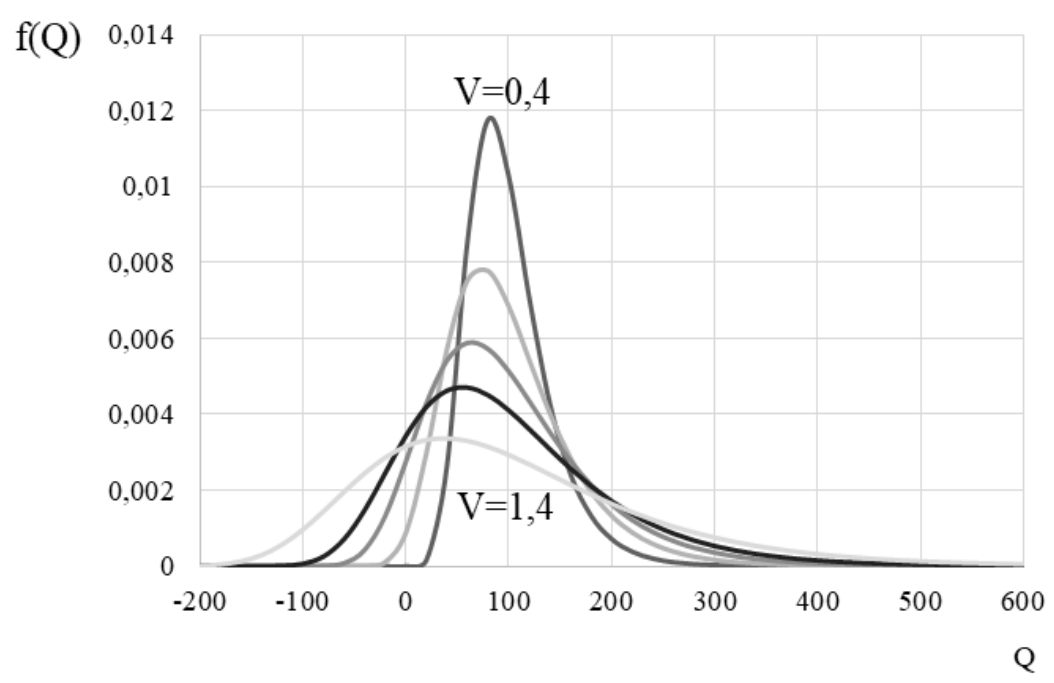

Figure 1 Probability density function of the Gumbel distribution with different values of the coefficient of variation

Table 1 Probability of negative values of random variable of Gumbel distribution

\begin{tabular}{cccccc}
\hline Coefficient of variation & $\mathbf{0 . 4}$ & $\mathbf{0 . 6}$ & $\mathbf{0 . 8}$ & $\mathbf{1 . 0}$ & $\mathbf{1 . 4}$ \\
\hline $\mathrm{F}(0)$ & 0.000001 & 0.0086 & 0.0615 & 0.1321 & 0.2458 \\
\hline
\end{tabular}

It is known that the coefficients of the variation of the annual snow maxima in areas with unstable snow cover can approach 1.0, and the coefficients of variation of the weight of ice can be much larger [2,13]. It can be seen from Fig. 1 and Table 1 that with such coefficients of variation, the probability of negative values becomes excessively high, potentially leading to significant errors in determining of the design values of these loads when using the Gumbel distribution.

The generalized extreme value distribution type-II [4] has an integral distribution function and probability density function, and can be written as follows:

$$
\begin{aligned}
& F(q)=\exp \left[-\left(\frac{q}{\beta}\right)^{-\alpha}\right] \\
& f(q)=\exp \left[-\left(\frac{q}{\beta}\right)^{-\alpha}\right] \times\left[\alpha\left(-\left(\frac{q}{\beta}\right)^{-\alpha-1}\right] / \beta\right.
\end{aligned}
$$

The parameters $\alpha$ and $\beta$ of the distribution in Equations (4) and (5) are determined according to recommendations [4], as follows: 


$$
M=\beta \cdot \Gamma(1-1 / \alpha), 1+V^{2}=\frac{\Gamma(1-2 / \alpha)}{\Gamma^{2}(1-/ \alpha)}
$$

In the above, $M$ is the mean value of the available data collection of maximum values; $V$ is the coefficient of variation of the available data collection of maximum values; and $\Gamma(n)$ is the Gamma function of the corresponding argument.

The dependence of the distribution on the coefficient of variation is shown in Fig. 2. The figure shows the curves of probability density function for the distribution of maxima of the second type (5) with the expected value $M=100$ and standard deviations $S=40,60,80,100$, and 140 , respectively. It can be seen from the figure that at close to zero values of the random variable, the probability density function of the distribution is practically zero. With an increase of the coefficient of variation, the distribution modal is shifted to the left and the probability density function on the right side increases, ensuring a corresponding increase of the standard deviation.

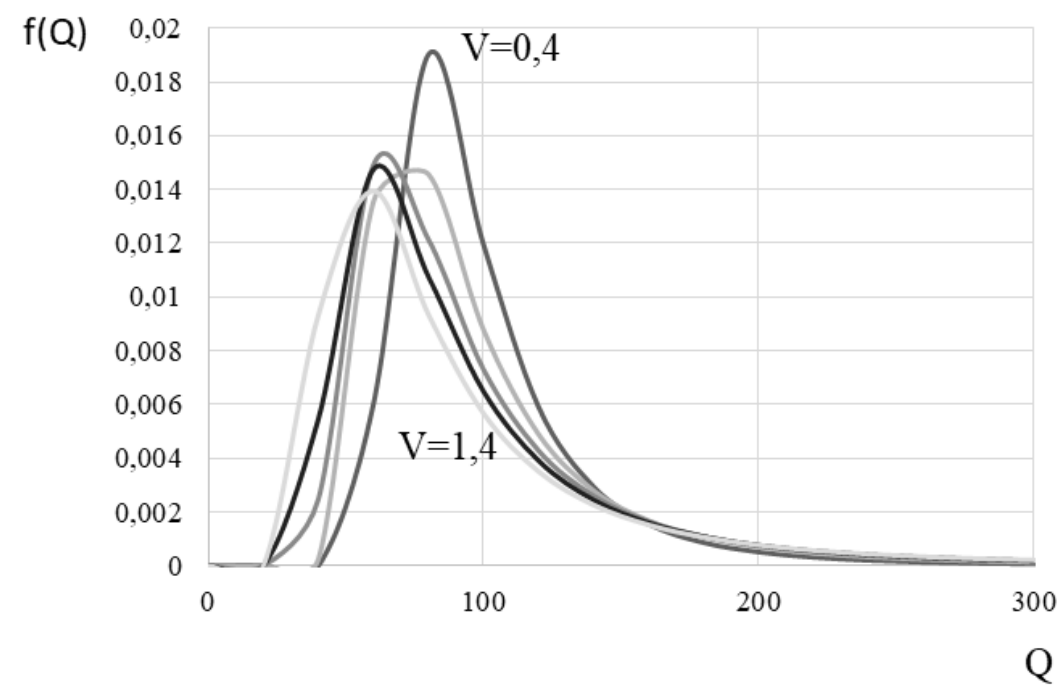

Figure 2 Probability density function of maxima of type-II distribution at different values of the coefficient of variation

The design load value corresponding to the return period $T$ is calculated as follows [4]:

$$
Q=\exp \left[\beta / \ln (1-1 / T)^{\frac{1}{\alpha}}\right]
$$

The advantage of the generalized extreme value distribution type-II (Equations (4) and (5)), which is a variant of the Fréchet distribution, is its positive definition area $q>0$, which corresponds to the physical nature of climatic loads.

The generalized extreme value distribution type-III $[3,4]$ is also called the Weibull distribution. The integral distribution function and probability density function of the extremums of the third type can be written as follows:

$$
\begin{aligned}
& (q)=1-\exp \left(-\beta \times x^{\alpha}\right) \\
& f(q)=\alpha \times \beta \times x^{\alpha-1} \times \exp \left(-\beta \times x^{\alpha}\right)
\end{aligned}
$$

According to the recommendations [2], the distribution parameters in Equations (8) and (9) are determined using corresponding equations, as follows: 


$$
M=\frac{\Gamma(1+1 / \alpha)^{\alpha}}{\beta}, 1+V^{2}=\frac{\Gamma(1+2 / \alpha)}{\Gamma^{2}(1+1 / \alpha)}
$$

The symbols in the equations (10) are similar to those in Equation (6).

The dependence of the form of the probability density function of the extremums of the third type (9) on the coefficient of variation is shown in Fig. 3 , where the curves of probability density function are shown for the same parameters as in Figs. 1 and 2.

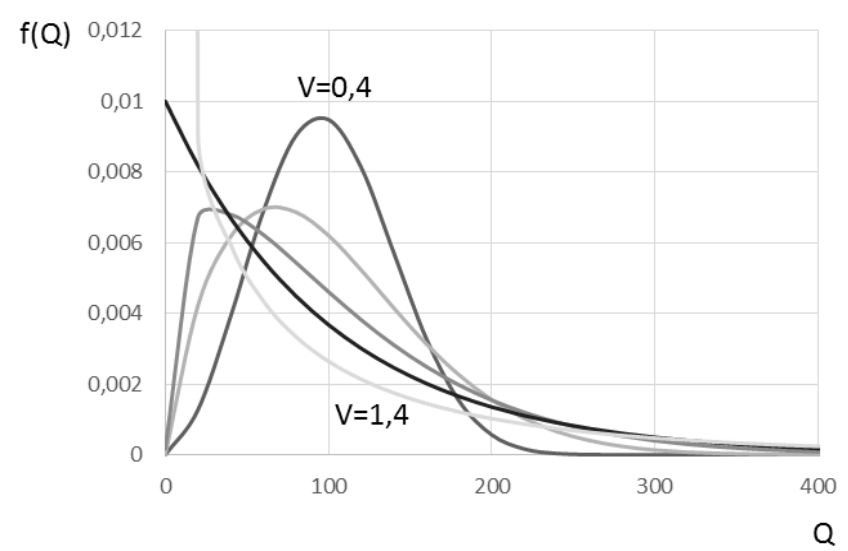

\section{Figure 3 Probability density function of type-III distribution with different values of the coefficient of} variation

From Fig. 3 , it can be seen that with coefficients of variation $V<1$, the probability density function has a hilllike appearance, and a larger $V$ means a more positive skew. At values of the coefficient of variation $V=0$, the distribution from Equations (8) and (9) transforms into an exponential distribution, and when $V>1$, the left part of the distribution curve increases to infinity, asymptotically approaching the $x$-axis. In all cases, the distribution from Equations (8) and (9) is defined in the region of positive numbers.

The design load value corresponding to the return period $T$ is calculated as follows [2]:

$$
Q=\left(\frac{[-\ln (1 / T)]}{\beta}\right)^{\frac{1}{\alpha}}
$$

The Weibull distribution is used not only for describing loads, but also for analyzing the strengths of materials and the carrying capacities of structures [14]. and (2).

The truncated Gumbel distribution is a modified version of the distribution of the first type, i.e., Equations (1)

To avoid negative load values, the domain of the function of the Gumbel distribution is restricted to zero, and the probability of implementing the negative values of $F(0)$ is considered on the right side of the distribution at $q>0$. The integral function and density of the truncated Gumbel distribution have the following form:

$$
\begin{aligned}
& F(q)=C \times \exp \left[-\exp \left(\frac{\alpha-q}{\beta}\right)\right]-C+1 \\
& f(q)=C \times \frac{1}{\beta} \exp \left[\frac{\alpha-Q}{\beta}-\exp \left(\frac{\alpha-q}{\beta}\right)\right]
\end{aligned}
$$


The coefficient $C \geq 1$ balances the truncated part of the distribution with $q<0$. The parameters of the truncated Gumbel distribution are determined by the method of moments $[4,15]$ as the roots of the nonlinear system, as follows:

$$
\left\{\begin{array}{l}
\int_{0}^{\infty} f(q) d q=1, \\
\int_{0}^{\infty} q \times f(q) d q=M, \\
\int_{0}^{\infty} q^{2} \times f(q) d q-M^{2}=S^{2},
\end{array}\right.
$$

where $f(q)$ is the probability density function (11); and $M$ and $S$ are the expected value and standard deviation of the distribution, respectively.

In the course of the study, it was found that the truncated Gumbel distribution (Equations (12) and (13)) can exist with coefficients of variation ranging from zero to one. The changes in the appearance of the probability density function of the truncated Gumbel distribution (13) with parameters (14) depending on its coefficient of variation within these limits are shown in Fig. 4.

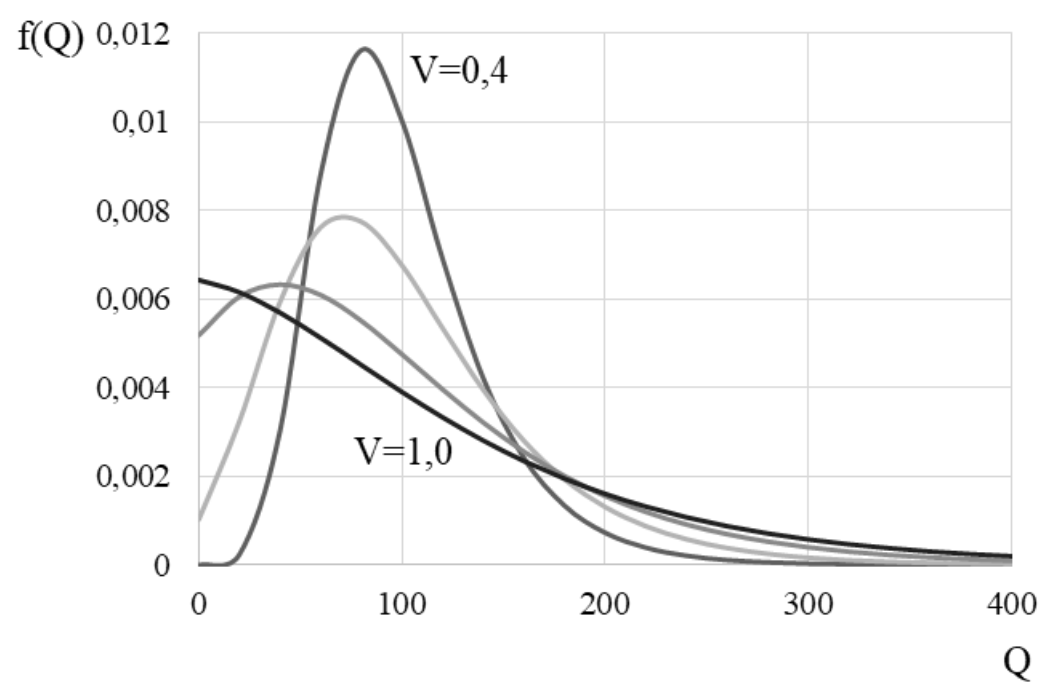

\section{Figure 4 Probability density function of the truncated Gumbel distribution for different values of the coefficient of variation}

It can be seen from the figure that an increase of the coefficient of variation leads to the enlargement of the region under the curve of probability density function and to the shifting of the distribution mode to the left, towards zero. When $V \rightarrow 1$, the mode takes a negative value and the right side of the curve of probability density function becomes more significant, i.e., resembling the exponential function with a coefficient of variation $V=1$. Thus, the truncated Gumbel distribution is unsuitable for describing the distributions of maximum load values with larger coefficients of variation (for example, the peaks of annual maxima of ice on some meteorological stations $[2,12]$ ).

The design load value corresponding to the return period $T$ is calculated as follows:

$$
Q(\mathrm{~T})=\alpha-\beta \cdot \ln \left[-\ln \left(\frac{T \cdot C-1}{T \cdot C}\right)\right]
$$


To compare the described distribution types, an analysis is conducted on their conformity with real results from meteorological observations, as well as a comparison of the design values of loads with different coefficients of variation for selected model examples.

\section{CONFORMITY OF EXTREME VALUE DISTRIBUTIONS WITH RESULTS OF METEOROLOGICAL OBSERVATIONS}

The suitability of the four described distribution types for the probabilistic description of climatic loads is analyzed using the Pearson's chi-squared test $[4,15]$. For this purpose, the results of meteorological observations from 20 meteorological stations uniformly distributed in different geographical regions of Ukraine were used. The network of weather stations is shown in Fig. 5.

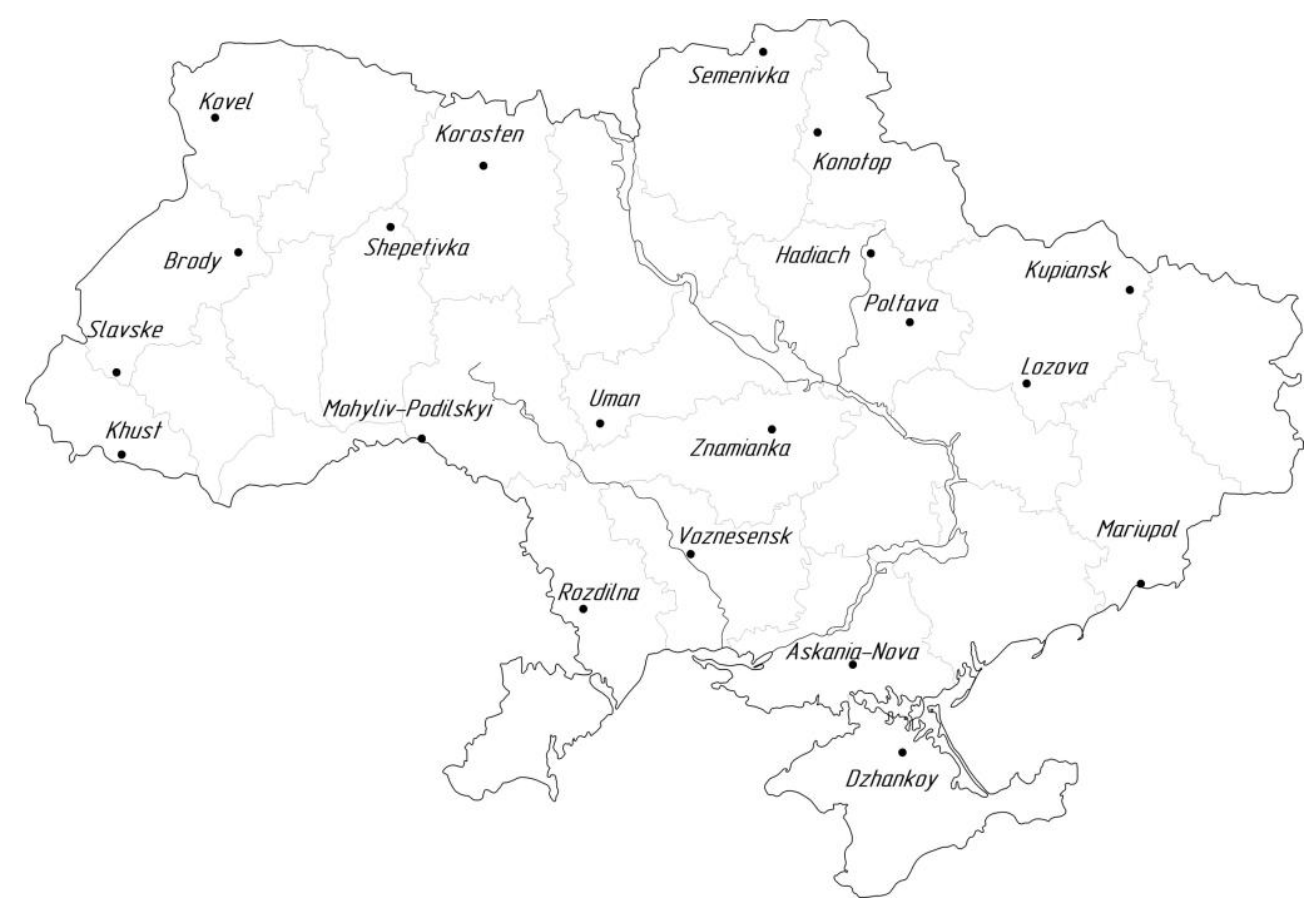

Figure 5 Scheme of selective network of meteorological stations in Ukraine

For the selected weather stations, the series of the wind and snow monitoring are quite long, allowing for analysis of the data collection for the annual maxima of the snow weight, wind speed, and pressure. In addition to checking the Pearson's chi-squared test, comparisons are made of the characteristic values of the investigated loads according to the different distribution types.

According to the results from the statistical processing of the data collection, it is found that the coefficient of variation of the annual snow weight maxima varies from 0.48 to 0.96 , that for the wind speed varies from 0.12 to 0.35 , and that for the wind pressure varies from 0.24 to 0.68 . Thus, the used data covers a wide range of coefficients of variation, and allows for general conclusions regarding the suitability of the various distribution types of extreme values.

The summary results from checking the conformity of the four distribution types with all of the 60 analyzed data collections are shown in Table 2 . The table contains the number of data elements that are not contradictory to the distribution types at the significance levels of $\alpha=0.05$ and $\alpha=0.01$. 
Table 2 Generalized results of checking the conformity of the distributions of the four types with data collection of annual maxima of climatic loads

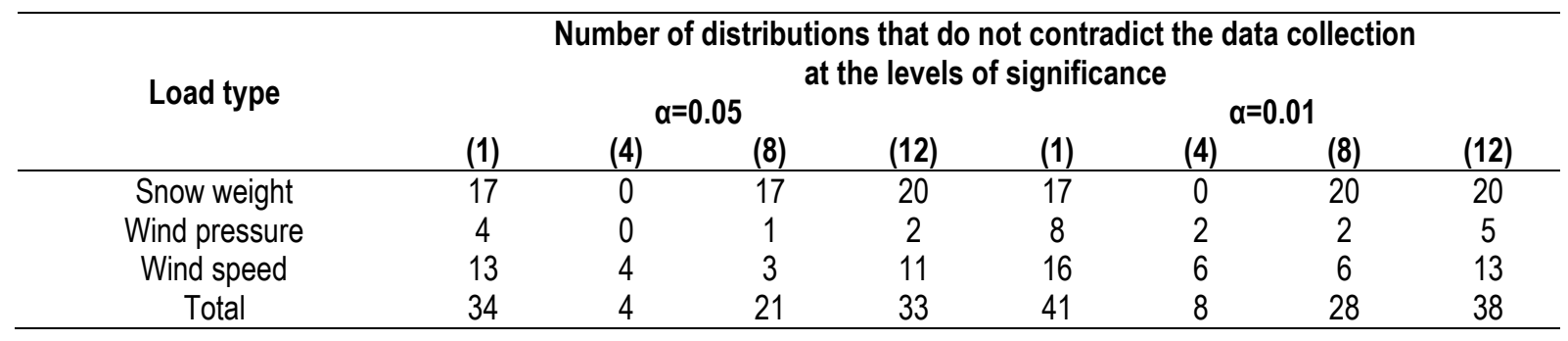

Analysis of Table 2 allows the following conclusions to be made regarding the conformity of the analyzed distribution types with the experimental data.

- The generalized extreme value distribution type-Il does not confirm with the experimental data in most cases, and therefore cannot be used for the probabilistic description of the annual maxima of climatic loads. Accordingly, it is not considered in further research.

- The other distribution types are much better suited to the data of the wind speed than to the data of the wind pressure.

- The generalized extreme value distribution type-I (Gumbel distribution) does not conform to the overwhelming majority of the data regarding the snow weight and wind speed at levels of significance of 0.05 and 0.01 .

- The generalized extreme value distribution type-III (Weibull distribution) is suitable for the description of data collections of the annual maxima of snow loads with large coefficients of variation, but is much worse for the wind speed data, which have lower values of coefficients of variation in general.

- The truncated Gumbel distribution is more suitable than the generalized extreme value distribution type-I for describing data collections regarding the snow weight.

The characteristic values of the loads $Q_{0}=Q(50)$ corresponding to a return period of 50 years can be calculated based on the four distribution types of extreme values using Equations (3), (7), (11), and (15). A comparative analysis shows that their differences do not exceed $18 \%$.

Based on the results of the analysis, the Gumbel distribution (type 1) and Weibull distribution (type 3) can be recommended for the probabilistic descriptions of the annual maxima of the snow weight and wind speed. The design values for the wind pressure are better determined based on the design values of the wind speed.

The following conclusions concern climatic loads that are typical for Europe, with the above-mentioned coefficients of variation in the samples of maximum values. Dramatically different climatic conditions can lead to slightly different distributions of the maximum values with other coefficients of variation. In this case, it is advisable to clarify the conclusions made by the analysis method herein, based on a sufficient amount of meteorological data.

\section{AREAS OF RATIONAL USE OF DISTRIBUTION TYPES OF EXTREME VALUES}

To identify areas for rational use of the Gumbel distribution (type 1) and Weibull distribution (type 3), the design values of loads as calculated using these distributions are compared for different return periods ranging from 20 to 1000 years. The comparison is made based on scientific modelling, and includes 10 distributions of annual maxima with the same expected values $M=100$ and coefficients of variation $0.2 \leq V \leq 2.0$.

For each of the ten values of the coefficient of variation, the parameters of the Gumbel (1) and Weibull (8) distributions are calculated, considering the actual size of the data collection of the annual maxima at $N=50$, i.e., for 50 years of meteorological observations. Considering the obtained parameters, the calculated load values for six return periods of $T=20,50,100,200,500$, and 1000 years are calculated according to Equations (3) and (11). The resulting tables for 60 design values (10 values of the coefficient of variation and six return periods) are compared with each other.

The fundamental impossibility of establishing the true distribution type leads to the choice of the distribution; nevertheless, the use of this choice allows for greater design load values, and provides a higher reliability level for the design of carrying structures. The criterion for choosing the distribution type is the ratio of the design values calculated by Equation (11) (based on the Weibull distribution) to the design values calculated by Equation (3)

Pashynskyi, M, Pashynskyi, V, Klymenko, E 
(based on the Gumbel distribution). This ratio reflects the percentage difference between the design load values as calculated by the two distribution types. The dependence of this ratio on the coefficient of variation of the data collection of the maxima and return period of the design values is shown in Fig. 6.

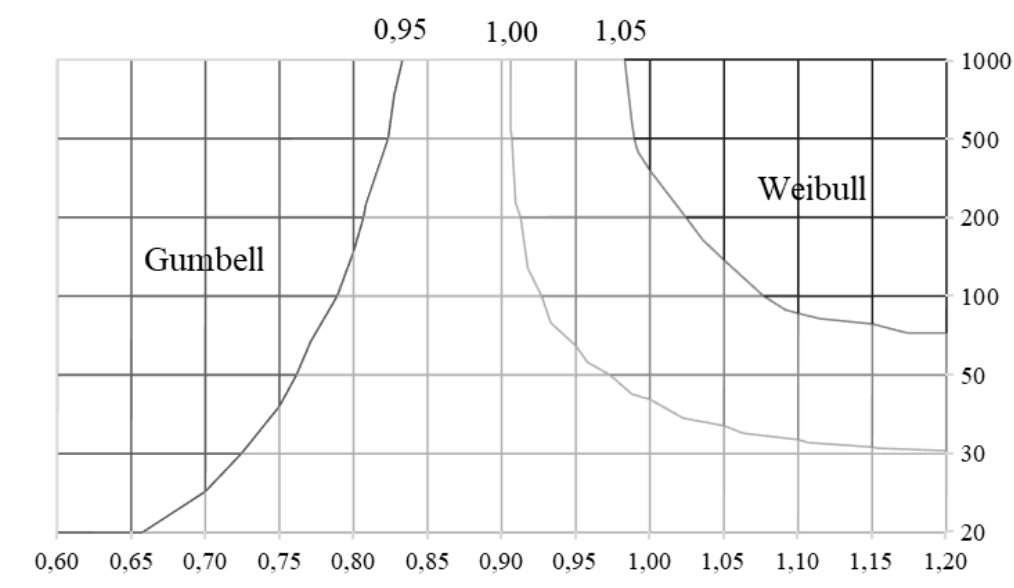

\section{Figure 6 Ratio of the design values of loads calculated by Weibull distribution to the design values calculated by Gumbel distribution}

The figure shows that the ratio mainly increases with increasing of coefficients of variation of the data collection and return periods of the design load values. A contour line with a value of 1.00 reflects combinations of return periods and coefficients of variation in which the use of both distribution types leads to identical load values. For ratios less than 0.95 (left side of the graph), it is better to use the Gumbel distribution, and for ratios greater than 1.05 (right zone of the graph), the Weibull distribution is more suitable. Within the contour lines of 0.95-1.05 with an error of no more than $\pm 5 \%$, it is possible to use both distribution types.

The dependence shown in Fig. 6 can be explained by the fact that at small values of the coefficient of variation, the Gumbel distribution gives fairly accurate results. The growth of the coefficient of variation leads to a lowering of the design values owing to the high probability of appearance of physically impossible negative load values. In these cases, it is better to use the Generalized extreme value distribution type-III (the Weibull distribution), which gives higher design values and therefore provides a higher reliability level.

A similar comparison within the coefficients of variation from 0.2 to 1.0 shows that the use of the truncated Gumbel distribution always leads to an increase in the design values of the loads relative to the original Gumbel distribution. With coefficients of variation up to 0.6 this increase is insignificant, but with further growth of the coefficient of variation and return period, it increases to $13 \%$. This can be explained by the increase of the probability of negative values using the Gumbel distribution, which are eliminated when using the truncated distribution.

This analysis allows to assert that for probabilistic descriptions of data collections of maxima with coefficients of variation less than $0.8-1.0$, the Gumbel distribution (1) should be used, and with larger coefficients of variation, the Weibull distribution (8) is preferable. Reducing the return periods of the design values extends the suitable area of use for the Gumbel distribution. It also becomes appropriate to use the Gumbel distribution when the value of the coefficient of variation exceeds 1.0 .

\section{ACCURACY OF EVALUATION OF CHARACTERISTIC VALUES OF CLIMATIC LOADS}

The characteristic and design load values can be calculated on the basis of various probabilistic models, the most common of which is the sequence of the annual maxima. The main characteristics of these models are determined by the results from the statistical processing of the data collection, making them random variables. Therefore, the design load values, as calculated based on random parameters of the probabilistic models, are also random, and have certain probable errors. 
The accuracy of the estimation of design values as calculated using different probabilistic models of climatic loads was analyzed in [2], where it was proposed to use a generalized accuracy characteristic in the form of a normalized error of design load, as follows:

$$
\varepsilon=\frac{\sqrt{N \times D_{Q}}}{Q_{0}},
$$

where $Q_{0}$ is the design value or characteristic value of the load; $N$ is the size of the data collection from which $Q_{0}$ was calculated; and $D_{Q}$ is the variance of data collection from which $Q_{0}$ was calculated.

According to the real meteorological data, the dependence of the standard deviation of the annual climatic load maxima of the data collection from its characteristic value, which is sufficiently accurate, that confirmed by the correlation coefficient of 0.995 was established.

$$
S=0,215 Q_{0},
$$

Based on the formula of the normalized error (16) of the design value given in [2], an approximate formula for the standard of the characteristic value is obtained as follows:

$$
S_{Q}=\frac{0,6 \times Q_{0}}{\sqrt{N}}
$$

where $Q_{0}$ is the characteristic value of load, and $N$ is the data collection of the annual maxima from which $Q_{0}$ was calculated.

Equation (18) considers the dependence in Equation (17) and the return period of the characteristic value as $T=50$ years. From Equation (18), it is easy to determine the probable relative error of the characteristic value, which, with a probability of 0.9 , will be calculated as follows:

$$
\Delta_{Q}= \pm \frac{100}{\sqrt{N}}(\%)
$$

where the standard deviation (18) and probable relative error (19) define the accuracy of the characteristic value, as obtained on the basis of the data collection of the annual maxima.

For example, the characteristic value of the climatic load calculated on the basis of a data collection of annual maxima with $N=20$ has a probable error of $\Delta Q=22 \%$. With 50 years of observation, the probable error is $\Delta Q=14$ $\%$, and with $N=100$ years, $\Delta Q=10 \%$. More precise formulas and nomograms for estimating the accuracy of the characteristic values of climatic loads are given in [15]. The use of Equation (19) and the previous data [15] allows for conclusions regarding the completeness of the meteorological information for a sufficiently accurate assessment of the characteristic values of climatic loads.

\section{CONCLUSIONS}

The generalized extreme value distribution type-I, which is traditionally used for the normalization of loads, has an area of negative values, and therefore can contradict the physical nature of loads in building constructions. It is shown that at large coefficients of variation, this can lead to errors in determining the design values of loads.

A comparative analysis of four distribution types of extreme values are made: the generalized extreme value distribution type-I, generalized extreme value distribution type-II, generalized extreme value distribution type-III, and truncated distribution of Gumbel. It is established that the truncated distribution of Gumbel exists only for coefficients of variation smaller than 1.0 .

Based on scientific modelling of the distribution types with different coefficients of variation in the range from 0.2 to 2.0, it is established that for probabilistic descriptions of annual load maxima with coefficients of variation less than $0.8-1.0$, it is recommended to use the Gumbel distribution. With larger values of the coefficients of variation, it is recommended to use the Weibull distribution. 
The accuracy of the estimation of the characteristic values according to the data collection of the annual load peaks is reflected in the standard deviation, which can be calculated using the characteristic value and data collection regarding the size of the annual maxima.

Further research is focused on clarifying the characteristic values of climatic loads for the territory of Ukraine, considering the results herein in terms of the rational choice of the distribution law and analysis of the accuracy of estimating characteristic values using samples of finite volume. The recommendations of this article can be used for the regulation of climatic loads in other countries.

\section{References}

[1] Verichev, K.; Carpio, M. 2018: Climatic zoning for building construction in a temperate climate of Chile, Sustainable cities and society, 40, pp. 352-364. https://doi.org/10.1016/j.scs.2018.04.020

[2] Pashynskyi, V. 1999: Atmospheric loads on building structures in Ukraine. UkrNDlproektstalkonstruktsiya, Kiev, Ukraine.

[3] EN 1991-1-3 (2003): Eurocode 1: Actions on structures - Part 1-3: General actions - Snow loads.

[4] Gumbel, E. J. 1958: Statistics of Extremes New York, Columbia University Press.

[5] Harris, R. 2001: The accuracy of design values predicted from extreme value analysis, Journal of Wind Engineering and Industrial Aerodynamics, 89 (2), pp. 153-164. https://doi.org/10.1016/S0167-6105(00)00060$\underline{X}$

[6] Forbes, C.; Evans, M.; Hastings, N.; Peacock, B. 2011: Statistical Distributions, 4th Edition, John Wiley \& Sons, Inc.

[7] Meløysund, V. et al. 2007: Effects of wind exposure on roof snow loads, Building and Environment, 42 (10) pp. 3726-3736. https://doi.org/10.1016/..buildenv.2006.09.005

[8] Walsh, A.; Costola, D.; Labaki, L. C. 2017: Review of methods for climatic zoning for building energy efficiency programs, Building and Environment, 112 (1), pp. 337-350. https://doi.org/10.1016/j.buildenv.2016.11.046

[9] Mauro, F.; Nabergoj R. 2017: An enhanced method for extreme loads analysis, Brodogradnja: Teorija i praksa brodogradnje i pomorske tehnike, 68 (2), pp. 79-92. http://dx.doi.org/10.21278/brod68206

[10] Jeromel, M.; Malačić, V.; Rakovec, J. 2009: Weibull distribution of bora and sirocco winds in the northern Adriatic Sea, Geofizika, 26 (1), pp. 85-100.

[11] Holicky, M.; Sykora, M. 2016: Probabilistic Models for Wind Actions, In 2016 Second International Symposium on Stochastic Models in Reliability Engineering, Life Science and Operations Management (SMRLO), pp. 172175. https://doi.org/10.1109/SMRLO.2016.38

[12] Kos, Ž.; Pashynskyi, V.; Klymenko, Y.; Pashynskyi, M. 2020: Analysis of Methods for Determining Climate Loads at a Specified Territory Point by Meteorological Data, Tehnički glasnik, 14 (2), pp. 206-211. https://doi.org/10.31803/tg-20191125075805

[13] Pashynskyi, M. 2017: Estimation accuracy of the calculated values of climatic loads, Modern engineering and innovative technologies, 2 (2). [in Ukrainian]

[14] Bužančić Primorac, B.; Parunov, J. 2015: Probabilistic Models of Ultimate Strength Reduction of Damaged Ship, Transactions of FAMENA, 39 (2), pp. 55-74.

[15] Seidenfeld, T. 2015: Probability Theory: Interpretations, International Encyclopedia of the Social \& Behavioral Sciences, pp. 37-42. https://doi.org/10.1016/B978-0-08-097086-8.42068-4

Please cite this article as: Pashynskyi, M., Pashynskyi, V., Klymenko, E.: Calculation of climate loads design values according to the probability model of annual maximum series, Electronic Journal of the Faculty of Civil Engineering Osijek-e-GFOS, 2021, 23, pp. 61-71, https://doi.org/10.13167/2021.23.6 